Proceedings of the Edinburgh Mathematical Society (2002) 45, 391-395 (C)

DOI:10.1017/S0013091500001061 Printed in the United Kingdom

\title{
ON $\omega$-INDEPENDENCE AND THE KUNEN-SHELAH PROPERTY
}

\author{
A. S. GRANERO ${ }^{1}$, M. JIMÉNEZ-SEVILLA ${ }^{1}$ AND J. P. MORENO ${ }^{2}$ \\ ${ }^{1}$ Departamento de Análisis Matemático, Facultad de Matemáticas, \\ Universidad Complutense de Madrid, 28040 Madrid, Spain \\ (granero@mat.ucm.es; marjim@mat.ucm.es) \\ ${ }^{2}$ Departamento de Matemáticas, Universidad Autonoma de Madrid, \\ 28049 Madrid, Spain (josepedro.moreno@uam.es)
}

(Received 2 November 2000)

\begin{abstract}
We prove that spaces with an uncountable $\omega$-independent family fail the Kunen-Shelah property. Actually, if $\left\{x_{i}\right\}_{i \in I}$ is an uncountable $\omega$-independent family, there exists an uncountable subset $J \subset I$ such that $x_{j} \notin \overline{\operatorname{conv}}\left(\left\{x_{i}\right\}_{i \in J \backslash\{j\}}\right)$ for every $j \in J$. This improves a previous result due to Sersouri, namely that every uncountable $\omega$-independent family contains a convex right-separated subfamily.
\end{abstract}

Keywords: $\omega$-independence; non-separable Banach spaces; Kunen-Shelah property

AMS 2000 Mathematics subject classification: Primary 46B20; 46B26

\section{Introduction}

Recently, two remarkable Banach spaces, one constructed by Kunen and another by Shelah, served as a source of counterexamples for a number of different problems. Shelah [10] was the first to construct a non-separable space satisfying what we called in $[\mathbf{3}]$ the Kunen-Shelah property: for every uncountable family $\left\{x_{i}\right\}_{i \in I}$, there is $i_{0} \in I$ such that

$$
x_{i_{0}} \in \overline{\operatorname{conv}}\left(\left\{x_{i}\right\}_{i \in I \backslash\left\{i_{0}\right\}}\right) .
$$

Spaces with the above property share, in a rather striking way, some of the features of separable spaces (see $[\mathbf{3}, \mathbf{4}, \mathbf{8}]$ ). The Shelah space was constructed assuming the diamond principle for $\omega_{1}$ and solved a problem by Davis and Johnson [9]. Later, assuming only the continuum hypothesis, Kunen $[\mathbf{6}]$ constructed a Banach space enjoying a stronger property: for every family $\left\{x_{\alpha}: \alpha<\omega_{1}\right\}$ there is $\alpha<\omega_{1}$ satisfying $x_{\alpha} \in{\overline{\left\{x_{\beta}: \alpha<\beta\right\}}}^{\text {weak }}$ (recall that a family $\left\{x_{\alpha}: \alpha<\omega_{1}\right\}$ in a topological space is right separated if $x_{\alpha} \notin \overline{\left\{x_{\beta}: \alpha<\beta\right\}}$ for all $\left.\alpha<\omega_{1}\right)$. The Kunen space is an Asplund $C(K)$ space with no Fréchet differentiable norms [4] and has many other interesting properties (see, for example, $[\mathbf{1}, \mathbf{3}]$ ).

Clearly, spaces with an uncountable biorthogonal system cannot satisfy the KunenShelah property. We encounter the notion of $\omega$-independence when looking for a weaker 
condition that ensures the failure of the above property. A family $\left\{x_{i}\right\}_{i \in I}$ is said to be $\omega$-independent if for every sequence $\left(i_{n}\right)_{n \geqslant 1} \subset I$ of distinct indices, and every sequence $\left(\lambda_{n}\right)_{n \geqslant 1} \subset \mathbb{R}$, the series $\sum_{n=1}^{\infty} \lambda_{n} x_{i_{n}}$ converges (in norm) to zero if and only if $\lambda_{n}=0$ for every $n \geqslant 1$ (see $[\mathbf{2}, \mathbf{5}]$ ). There are $\omega$-independent families which are not biorthogonal systems. Here is one example: $X=C\left([0,1]^{\omega_{1}}\right)$ and $\left\{f_{\alpha}^{n}\right\}_{\alpha<\omega_{1}, n \in \mathbb{N}}$ defined as

$$
f_{\alpha}^{n}\left(\left(t_{\gamma}\right)_{\gamma<\omega_{1}}\right)=t_{\alpha}^{n}
$$

for every $x=\left(t_{\gamma}\right)_{\gamma<\omega_{1}} \in[0,1]^{\omega_{1}}$. The purpose of this paper is to prove that spaces with the Kunen-Shelah property contain no uncountable $\omega$-independent families.

Unless otherwise stated, by a family we always understand an uncountable set, so we often omit this adjective. Let us say that the family $F=\left\{x_{\alpha}: \alpha<\omega_{1}\right\}$ has the Kunen-Shelah property if among any $\omega_{1}$ elements of $F$ there is one that belongs to the closed convex hull of the rest (i.e. the space has the Kunen-Shelah property if and only if every uncountable family also has). The family $F$ is said to be convex right separated if $x_{\alpha} \notin \overline{\operatorname{conv}}\left(\left\{x_{\beta}\right\}_{\beta>\alpha}\right)$ for every $\alpha<\omega_{1}$. Finally, the family $\left\{x_{i}\right\}_{i \in I}$ is polyhedral provided

$$
x_{j} \notin \overline{\operatorname{conv}}\left(\left\{x_{i}\right\}_{i \in I \backslash\{j\}}\right)
$$

for every $j \in I$. Sersouri [8] proved that an $\omega$-independent family always contains a convex right-separated family. We will improve his result by showing that an $\omega$-independent family always contains a polyhedral subfamily. As a consequence, $\omega$-independent families never have the Kunen-Shelah property.

\section{Uncountable $\omega$-independent families fail the Kunen-Shelah property}

The Sersouri proof, as ours, is based on the following result due to Kalton [5]. Let $\left(a_{n}\right)_{n \geqslant 1}$ be a sequence of positive real numbers such that $\lim _{n} a_{n}=0$ and $\sum_{n \geqslant 1} a_{n}=\infty$. Let $b_{n}=\sup _{m \geqslant n} a_{m}$. If $\left\{x_{\alpha}: \alpha<\omega_{1}\right\}$ is an uncountable family of norm-one elements, then for every $x \in \operatorname{span}\left\{x_{\alpha}: \alpha<\omega_{1}\right\}$, every $\delta>0$ and every $n \in \mathbb{N}$ there exist $m>n$, a sequence of signs $\left\{\varepsilon_{i}\right\}_{i=n+1}^{m}$ and a sequence of (not necessarily distinct) ordinals $\left\{\alpha_{i}\right\}_{i=n+1}^{m}$ such that

$$
\left\|x+\sum_{i=n+1}^{m} a_{i} \varepsilon_{i} x_{\alpha_{i}}\right\|<\delta
$$

and

$$
\sup _{n<k<m}\left\|x+\sum_{i=n+1}^{k} a_{i} \varepsilon_{i} x_{\alpha_{i}}\right\|<\|x\|+\delta+b_{n} .
$$

Taking a subfamily if necessary, every $x_{\alpha}$ is an accumulation point of $\left\{x_{\alpha}: \alpha<\omega_{1}\right\}$ when the space is separable. Using this fact and the above two conditions, Kalton constructs inductively a series $\sum a_{j} \varepsilon_{j} x_{\alpha_{j}}$ convergent to zero with $\left\{\alpha_{j}\right\}$ different ordinals. In order to apply the Kalton technique in the Kunen space, Sersouri first showed that it can be assumed that $\overline{\operatorname{conv}}\left\{x_{\alpha}: \alpha<\omega_{1}\right\}=\overline{\operatorname{conv}}\left\{x_{\alpha}: \beta<\alpha<\omega_{1}\right\}$ for every $\beta<\omega_{1}$ and, consequently, that every $x_{\gamma}$ is an accumulation point of $\operatorname{conv}\left\{x_{\alpha}: \beta<\alpha<\omega_{1}\right\}$. In our case, a preparatory lemma together with the Kalton construction are the key tools of the proof. 
Lemma 2.1. If a convex right-separated family $\left\{y_{\alpha}: \alpha<\omega_{1}\right\}$ satisfies the KunenShelah property, there exists a subfamily $\left\{x_{\alpha}: \alpha<\omega_{1}\right\} \subset\left\{y_{\alpha}: \alpha<\omega_{1}\right\}$, an ordinal $\beta_{0}<\omega_{1}$ and a positive number $\varepsilon_{0}$ such that every $x_{i}$, with $\beta_{0} \leqslant i<\omega_{1}$, is an accumulation point of $\left[0,1+1 / \varepsilon_{0}\right] D_{\gamma}-\left[0,1 / \varepsilon_{0}\right] D_{\gamma}$, for every $\gamma<\omega_{1}$ and $D_{\gamma}=\operatorname{conv}\left(\left\{x_{\alpha}\right\}_{\gamma<\alpha}\right)$.

Proof. Assume that $\left\|y_{\alpha}\right\| \leqslant 1$ for every $\alpha<\omega_{1}$. Since the family $\left\{y_{\alpha}: \alpha<\omega_{1}\right\}$ is convex right separated, we can choose a subfamily $\left\{x_{\alpha}: \alpha<\omega_{1}\right\} \subset\left\{y_{\alpha}: \alpha<\omega_{1}\right\}$ and a positive number $\varepsilon_{0}>0$ satisfying

$$
\operatorname{dist}\left(x_{\alpha}, \overline{\operatorname{conv}}\left(\left\{x_{\beta}\right\}_{\beta>\alpha}\right)\right) \geqslant 3 \varepsilon_{0}
$$

for every $\alpha<\omega_{1}$. Let $n_{0} \in \mathbb{N}$ be such that $1 / n_{0}<\varepsilon_{0}$. Define $A$ to be the subset of all ordinals $\alpha<\omega_{1}$ for which there are $\alpha<\rho<\gamma<\omega_{1}$ such that

$$
x_{\rho} \notin \overline{\operatorname{conv}}\left(\left\{x_{\beta}\right\}_{\beta \leqslant \alpha} \cup\left\{x_{\beta}\right\}_{\gamma \leqslant \beta<\omega_{1}}\right) .
$$

Since $\left\{x_{\alpha}: \alpha<\omega_{1}\right\}$ satisfies the Kunen-Shelah property, $A$ must be countable. Let $\tau=\sup \{\alpha: \alpha \in A\}$ and $B=\overline{\operatorname{conv}}\left(\left\{x_{\beta}\right\}_{\beta \leqslant \tau}\right)$. It is clear that

$$
x_{\rho} \in \overline{\operatorname{conv}}\left(B \cup D_{\gamma}\right)
$$

whenever $\tau<\rho<\gamma<\omega_{1}$. Now define $B(\rho, \gamma, n)$ to be the subset of $B$ of all $u$ with the property that there is $v \in D_{\gamma}$ and $\lambda \in(0,1]$ with

$$
\left\|\lambda u+(1-\lambda) v-x_{\rho}\right\|<1 /(2 n) .
$$

Note first that $B(\rho, \gamma, n) \neq \emptyset$ if $n>n_{0}$. By definition, $B(\rho, \gamma, n+1) \subset B(\rho, \gamma, n)$ and $B\left(\rho, \gamma^{\prime}, n\right) \subset B(\rho, \gamma, n)$ for every $\gamma^{\prime}>\gamma$. If $u, v, \lambda$ satisfy the conditions of $B(\rho, \gamma, n)$ with $n>n_{0}$, then (2.1) implies that

$$
2 \lambda \geqslant\|\lambda(u-v)\|>3 \varepsilon_{0}-(1 /(2 n))>2 \varepsilon_{0} ;
$$

so, in the definition of $B(\rho, \gamma, n)$ we can write $\lambda \in\left(\varepsilon_{0}, 1\right]$ instead of $\lambda \in(0,1]$. Given $\tau \leqslant \beta<\omega_{1}$ and $n \geqslant n_{0}$, define $B(\beta, n)$ as the closure of the union of all $B(\rho, \gamma, n)$ with $\beta \leqslant \rho<\gamma<\omega_{1}$. Again, $B(\beta, n+1) \subset B(\beta, n)$ and $B\left(\beta^{\prime}, n\right) \subset B(\beta, n)$ if $\tau \leqslant \beta<\beta^{\prime}$, $B(\beta, n) \neq \emptyset$ if $n \geqslant n_{0}$.

Since $B$ is hereditarily Lindelöff (it is separable metric complete), for each $n \geqslant n_{0}$ there exists $\tau \leqslant \beta_{n}<\omega_{1}$ such that for every $\beta_{n} \leqslant \beta<\omega_{1}$ we have $B(\beta, n)=B\left(\beta_{n}, n\right)$. Let $\beta_{0}=\sup _{n} \beta_{n}$ and fix $\beta_{0} \leqslant \rho<\gamma<\omega_{1}, n \geqslant n_{0}$. Pick $u \in B(\rho, \gamma, n), \mu \in\left(\varepsilon_{0}, 1\right]$ and $\omega \in D_{\gamma}$ such that $\left\|(\mu u+(1-\mu) w)-x_{\rho}\right\|<1 /(2 n)$. Since $u \in B(\gamma, n)$ also, there exist $\gamma \leqslant \sigma<\theta<\omega_{1}, \lambda \in\left(\varepsilon_{0}, 1\right]$ and $v \in D_{\theta}$ such that $\left\|(\lambda u+(1-\lambda) v)-x_{\sigma}\right\|<1 /(2 n)$. Letting $y=x_{\sigma}-(\lambda u+(1-\lambda) v)$, we have $u=\left(x_{\sigma}-y-(1-\lambda) v\right) / \lambda$ and

$$
\left\|\mu \frac{x_{\sigma}-y-(1-\lambda) v}{\lambda}+(1-\mu) w-x_{\rho}\right\|<\frac{1}{2 n} .
$$


Now, taking into account that $0<\mu / \lambda<1 / \varepsilon_{0}$ and $\|y\|<1 /(2 n)$, we obtain

$$
\begin{aligned}
\left\|\mu \frac{x_{\sigma}-(1-\lambda) v}{\lambda}+(1-\mu) w-x_{\rho}\right\| & <\frac{1}{2 n}+\frac{\mu}{\lambda}\|y\| \\
& <\frac{1}{2 n}+\frac{1}{2 n} \frac{1}{\varepsilon_{0}}=\frac{1}{2 n}\left(1+\frac{1}{\varepsilon_{0}}\right) .
\end{aligned}
$$

which implies that $x_{\rho} \in E_{\gamma}:=\overline{\left[\left\{x_{i}\right\}_{\gamma \leqslant i<\omega_{1}}\right]}$, since $x_{\sigma}, v, w \in E_{\gamma}$ and $n>n_{0}$ is arbitrary. In particular, it means that $E_{\beta_{0}}=E_{\beta}$ for every $\beta_{0} \leqslant \beta<\omega_{1}$. Finally, if we denote $z=x_{\rho}-\left[(\mu / \lambda)\left(x_{\sigma}-y-(1-\lambda) v\right)+(1-\mu) w\right]$, then

$$
x_{\rho}=\left(\mu v+(1-\mu) w+\frac{\mu}{\lambda} x_{\sigma}\right)-\frac{\mu}{\lambda} v+z,
$$

thus implying that $x_{\rho}$ is an accumulation point of

$$
\left[0,1+\frac{1}{\varepsilon_{0}}\right] D_{\gamma}-\left[0, \frac{1}{\varepsilon_{0}}\right] D_{\gamma}=F_{\gamma}
$$

Indeed, $0<\mu / \lambda<1 / \varepsilon_{0}, \mu v+(1-\mu) w, x_{\sigma}, v \in D_{\gamma}$ and $\|z\|<1 /(2 n)\left(1+\left(1 / \varepsilon_{0}\right)\right)$.

Theorem 2.2. Every uncountable $\omega$-independent family fails the Kunen-Shelah property. Consequently, spaces with the Kunen-Shelah property have no w-independent families.

Proof. By the Sersouri result, we know that an uncountable $\omega$-independent family $F=\left\{x_{\alpha}: \alpha<\omega_{1}\right\}$ always contains a convex right-separated family, so we may assume that $F$ is convex right separated. By Lemma 2.1 (choosing a subfamily, if necessary) we can also suppose the existence of $\beta_{0}<\omega_{1}$ and $\varepsilon_{0}>0$ so that every $x_{i}$, with $\beta_{0} \leqslant i<\omega_{1}$, is an accumulation point of $\left[0,1+1 / \varepsilon_{0}\right] D_{\gamma}-\left[0,1 / \varepsilon_{0}\right] D_{\gamma}$, for every $\gamma<\omega_{1}$. Pick $x_{j} \in$ $F$ with $\beta_{0}<j$. As in [5], we can construct inductively a sequence of signs $\left(\varepsilon_{n}\right)_{n \geqslant 1}$, a sequence of positive real numbers $\left\{\lambda_{p}^{n}, \mu_{p}^{n}\right\}_{n \geqslant 1,1 \leqslant p \leqslant k(n)}$ and a sequence of ordinals $\left(\gamma_{p}^{n}\right)_{n \geqslant 1,1 \leqslant p \leqslant k(n)}$ such that

$$
\begin{array}{ll}
\text { (a) } \sum_{p=1}^{k(n)} \lambda_{p}^{n} \in\left[0,1+\frac{1}{\varepsilon_{0}}\right], \sum_{p=1}^{k(n)} \mu_{p}^{n} \in\left[0, \frac{1}{\varepsilon_{0}}\right] \quad \text { for every } n \geqslant 1, \\
\text { (b) } \beta_{0}<j<\gamma_{1}^{n}<\gamma_{2}^{n}<\cdots<\gamma_{k(n)}^{n}<\gamma_{1}^{n+1}<\omega_{1} \quad \text { for every } n \geqslant 1,
\end{array}
$$

and

$$
x_{j}+\sum_{n \geqslant 1} a_{n} \varepsilon_{n} y_{n}=0
$$

where $y_{n}=\sum_{p=1}^{k(n)}\left(\lambda_{p}^{n}-\mu_{p}^{n}\right) x_{\gamma_{p}^{n}}$. Now it is easy to see that the series

$$
x_{j}+\sum_{n \geqslant 1} a_{n} \varepsilon_{n}\left(\sum_{p=1}^{k(n)}\left(\lambda_{p}^{n}-\mu_{p}^{n}\right) x_{\gamma_{p}^{n}}\right)
$$

also converges to zero, thus proving that $\left\{x_{i}\right\}_{i<\omega_{1}}$ is not $\omega$-independent, a contradiction. 


\section{Remarks}

An Asplund space $X$ with the Kunen-Shelah property has no convex right-separated families. Indeed, every weak ${ }^{*}$ compact convex subset of $X^{*}$ is the weak* closed convex hull of its weak* strongly exposed points [7]. As shown in [4], the set of weak* denting points of the dual unit ball of a Banach space with the Kunen-Shelah property lies in a separable subspace. Consequently, $X^{*}$ is weak* separable and then $X$ cannot contain a convex right-separated family $\left\{x_{\alpha}: \alpha<\omega_{1}\right\}$. Otherwise, the nested sequence $\left\{C_{\beta}=\overline{\operatorname{conv}}\left(\left\{x_{\alpha}\right\}_{\beta<\alpha}\right), \beta<\omega_{1}\right\}$ would produce, by duality, an (uncountable) nested sequence of different weak* closed convex sets in $X^{*}$.

In general, the Kunen-Shelah property seems to be a weaker condition than the absence of convex right-separated families. However, there is no example of a Banach space with the Kunen-Shelah property admitting such a family. The Kunen-Shelah property suffices for most purposes and it can be connected with many usual geometrical features of Banach spaces.

Let us finish this paper with an open problem. It is natural to ask if the condition $\overline{\operatorname{conv}}\left\{x_{\alpha}: \beta_{0} \leqslant \alpha<\omega_{1}\right\}=\overline{\operatorname{conv}}\left\{x_{\alpha}: \beta \leqslant \alpha<\omega_{1}\right\}$ for every $\beta_{0} \leqslant \beta<\omega_{1}$, used by Sersouri, and the condition $x_{i} \in \overline{\left[0,1+1 / \varepsilon_{0}\right] D_{\gamma}-\left[0,1 / \varepsilon_{0}\right] D_{\gamma}}$ for every $\beta_{0}<i<\omega_{1}$, and every $\gamma<\omega_{1}$, used in our proof, can be replaced by other stationary conditions. For instance, is it true that a family $\left\{x_{\alpha}: \alpha<\omega_{1}\right\}$ satisfying

$$
\overline{\operatorname{span}}\left\{x_{\alpha}: \beta_{0} \leqslant \alpha<\omega_{1}\right\}=\overline{\operatorname{span}}\left\{x_{\alpha}: \beta \leqslant \alpha<\omega_{1}\right\}
$$

for every $\beta_{0} \leqslant \beta<\omega_{1}$ cannot be $\omega$-independent?

Acknowledgements. Supported in part by DGICYT grants PB 97-0240 and BMF 2000-0609.

\section{References}

1. C. Finet and G. Godefroy, Biorthogonal systems and big quotient spaces, Contemp. Math. 85 (1989), 87-110.

2. D. H. Fremlin And A. Sersouri, On $\omega$-independence in separable Banach spaces, $Q$. J. Math. 39 (1988), 323-331.

3. A. S. Granero, M. Jiménez-Sevilla and J. P. Moreno, Convex sets in Banach spaces and a problem of Rolewicz, Studia Math. 129 (1998), 19-29.

4. M. Jiménez-Sevilla And J. P. Moreno, Renorming Banach spaces with the Mazur intersection property, J. Funct. Analysis 144 (1997), 486-504.

5. N. J. Kalton, Independence in separable Banach spaces, Contemp. Math. 85 (1989), 319-323.

6. S. NeGrepontis, Banach spaces and topology, in Handbook of set-theoretic topology, pp. 1045-1142 (North-Holland, Amsterdam, 1984).

7. R. R. Phelps, Convex functions, monotone operators and differentiability, 2nd edn, Lecture Notes in Mathematics, no. 1364 (Springer, 1993).

8. A. Sersouri, $\omega$-independence in nonseparable Banach spaces, Contemp. Math. 85 (1989), 509-512.

9. S. Shelah, A Banach space with few operators, Israel J. Math. 30 (1978), 181-191.

10. S. Shelah, Uncountable constructions for B.A., e.c. groups and Banach spaces, Israel J. Math. 51 (1985), 273-297. 